\title{
Properties of Coefficients of Certain Linear Forms in Generalized Polylogarithms.
}

\author{
Zlobin S.A.
}

August 30, 2018 
UDK 511.36

Abstract. We study properties of coefficients of a linear form, originating from a multiple integral. As a corollary, we prove Vasilyev's conjecture, connected with the problem of irrationality of the Riemann zeta function at odd integers.

Keywords: irrationality, Riemann zeta function, Vasiliev's conjecture, generalized polylogarithm, multiple integral, linear form, denominators, coefficients estimate.

\section{Introduction}

Define a generalized polylogarithm by the series

$$
\operatorname{Le}_{\vec{s}}(z)=\sum_{n_{1} \geq n_{2} \geq \cdots \geq n_{l} \geq 1} \frac{z^{n_{1}}}{n_{1}^{s_{1}} n_{2}^{s_{2}} \ldots n_{l}^{s_{l}}}
$$

for a vector $\vec{s}=\left(s_{1}, \ldots, s_{l}\right), s_{j} \in \mathbb{N}$. This series converges when $|z|<1$. In the paper [1], in connection with approximations of values of generalized polylogarithms, it has been proved the following result. Let $a_{i}, b_{i}, c_{j}$ be integers, satisfying the certain inequalities. Then, the identity

$$
S(z)=\int_{[0,1]^{m}} \frac{\prod_{i=1}^{m} x_{i}^{a_{i}-1}\left(1-x_{i}\right)^{b_{i}-a_{i}-1}}{\prod_{j=1}^{l}\left(1-z x_{1} x_{2} \ldots x_{r_{j}}\right)^{c_{j}}} d x_{1} d x_{2} \ldots d x_{m}=\sum_{\vec{s}} P_{\vec{s}}\left(z^{-1}\right) \operatorname{Le}_{\vec{s}}(z),
$$

holds, where $0=r_{0}<r_{1}<r_{2}<\cdots<r_{l}=m$ and $P_{\vec{s}}$ are polynomials with rational coefficients. This representation is unique because of the linear independence of $\operatorname{Le}_{\vec{s}}(z)$ with different indices over $\mathbb{C}(z)$ (see [1, Corollary 1]).

It is important in arithmetical applications to have estimate for absolute values and a common denominator of coefficients of the polynomials $P_{\vec{s}}$ depending on the parameters $a_{i}, b_{i}, c_{j}$, as well as on the dimension $m$ of the integral. This is the main aim of this paper.

One of possible applications of integrals of type $S(z)$ is related to the problem of the irrationality of the Riemann zeta function $\zeta(k)$ at odd integers 
$k=3,5,7, \ldots$ In [2], D.V. Vasilyev considered the integrals

$$
V_{m, n}=\int_{[0,1]^{m}} \frac{\prod_{i=1}^{m} x_{i}^{n}\left(1-x_{i}\right)^{n}}{\left(1-x_{1}\left(1-x_{2}\left(\cdots-x_{m-1}\left(1-x_{m}\right) \cdots\right)^{n+1}\right.\right.} d x_{1} d x_{2} \ldots d x_{m} .
$$

He conjectered that

$$
V_{2 l+1, n}=A_{0}+\sum_{j=1}^{l} A_{j} \zeta(2 j+1), \quad D_{n}^{2 l+1} A_{j} \in \mathbb{Z},
$$

where $D_{n}$ is the least common multiple of $1,2, \ldots, n$. The integral $V_{3, n}$ is equal to the integral, which was used by F. Beukers for the proof of the irrationality of $\zeta(3)$ (see [3]). The equality (2) holds for it. Vasilyev proved (2) for $m=5$. Later W.V. Zudilin ([4]) showed (2) with the weaker inclusion $D_{n}^{2 l+2} \Phi_{n}^{-1} A_{j} \in \mathbb{Z}$, where $\Phi_{n}$ is the product of prime numbers $p<n$ for which $2 / 3 \leq\{n / p\}<1(\{\cdot\}$ denotes the fractional part of a number). The validity of $D_{n}^{2 l+1} A_{j} \in \mathbb{Z}$ was proved by C. Krattenthaler and T. Rivoal ([프, Théorème 1]). Their proof is technically complicated. In this paper we prove (2) using the following representation $V_{m, n}$ in the form (11) (see [6, Corollary 2]):

$$
V_{2 l+1, n}=\int_{[0,1]^{2 l+1}} \frac{\prod_{i=1}^{2 l+1} x_{i}^{n}\left(1-x_{i}\right)^{n} d x_{1} d x_{2} \ldots d x_{2 l+1}}{\prod_{j=1}^{l}\left(1-x_{1} \ldots x_{2 j}\right)^{n+1}\left(1-x_{1} x_{2} \ldots x_{2 l} x_{2 l+1}\right)^{n+1}} .
$$

We prove theorems 1 and 2 in sections 3 and 4 of this article. They give the estimate on the common denominator and the values of the coefficients $P_{\vec{s}}$ in (11) under the certain conditions.

\section{Elementary Sums}

We call a sum of type

$$
\sum_{n_{1} \geq n_{2} \geq \cdots \geq n_{l} \geq 1} z^{n_{1}-1} \prod_{j=1}^{l} \frac{1}{\left(n_{j}+p_{j}\right)^{u_{j}}}, \quad p_{j} \in\{0,1,2, \ldots\}, \quad u_{j} \in \mathbb{N},
$$

elementary. From [1, Theorem 1] it follows that this sum can be expressed in the form (11). 
In what follows, for any vector $\vec{s}=\left(s_{1}, s_{2}, \ldots, s_{l}\right)$ we use the notation $w(\vec{s})=s_{1}+s_{2}+\cdots+s_{l}$. The height of the polynomial is the maximum of the absolute values of its coefficients.

Lemma 1 Let $P=\max _{1 \leq j \leq l} p_{j}$. Then, for the sum (4), the heights of the polynomials $P_{\vec{s}}$ do not exceed

$$
\max \left(l ! \cdot\left(w(\vec{u}) 2^{w(\vec{u})}\right)^{l-1} P^{l}, 1\right)
$$

moreover, $D_{P}^{w(\vec{u})-w(\vec{s})} P_{\vec{s}}(z) \in \mathbb{Z}[z]$.

Proof. We use the following notation: $r_{0}=0, r_{j}=u_{1}+u_{2}+\cdots+u_{j}$, $m=r_{l}=w(\vec{u})$. By [1, Lemma 2] it is possible to write expression (4) as the integral

$$
I\left(p_{1}, p_{2}, \ldots, p_{l}\right)=\int_{[0,1]^{m}} \frac{\prod_{j=1}^{l}\left(x_{r_{j-1}+1} x_{r_{j-1}+2} \ldots x_{r_{j}}\right)^{p_{j}}}{\prod_{j=1}^{l}\left(1-z x_{1} x_{2} \ldots x_{r_{j}}\right)} d x_{1} d x_{2} \ldots d x_{m} .
$$

We prove Lemma 1 by induction on the vector $\left(l, p_{1}+p_{2}+\cdots+p_{l}\right)$. We order vectors $(l, k)$ in lexicographic ally, i.e.

$$
\left(l_{1}, k_{1}\right)<\left(l_{2}, k_{2}\right) \Leftrightarrow l_{1}<l_{2} \text { or } l_{1}=l_{2} \text { and } k_{1}<k_{2} \text {. }
$$

The statement, which is proved by induction, is a little stricter than the statement of the lemma: the heights of $P_{\vec{s}}(z)$ do not exceed

$$
\max \left(\sum_{j=1}^{l} p_{j} \cdot(l-1) ! \cdot\left(m 2^{m} P\right)^{l-1}, 1\right) .
$$

This estimate is really more precise than (5) since $\sum_{j=1}^{l} p_{j} \leq l \cdot P$. The induction base $\left(p_{1}=p_{2}=\cdots=p_{l}=0\right)$ follows from (44): $I(0,0, \ldots, 0)=$ $z^{-1} \operatorname{Le}_{u_{1}, u_{2}, \ldots, u_{l}}(z)$.

Let $p_{h}>0$ for some $h>1$. From the equality

$$
\begin{aligned}
\left(x_{r_{h-1}+1} x_{r_{h-1}+2} \ldots x_{r_{h}}\right)^{p_{h}}= & \left(x_{r_{h-1}+1} x_{r_{h-1}+2} \ldots x_{r_{h}}\right)^{p_{h}-1} \\
& +\left(x_{r_{h-1}+1} x_{r_{h-1}+2} \ldots x_{r_{h}}\right)^{p_{h}}\left(1-z x_{1} x_{2} \ldots x_{r_{h-1}}\right)
\end{aligned}
$$




$$
-\left(x_{r_{h-1}+1} x_{r_{h-1}+2} \ldots x_{r_{h}}\right)^{p_{h}-1}\left(1-z x_{1} x_{2} \ldots x_{r_{h}}\right)
$$

it follows that

$$
\begin{aligned}
I\left(p_{1}, p_{2}, \ldots, p_{h}, \ldots, p_{l}\right)= & I\left(p_{1}, p_{2}, \ldots, p_{h}-1, \ldots, p_{l}\right) \\
& +\int_{[0,1]^{m}} \frac{\prod_{j=1}^{l}\left(x_{r_{j-1}+1} x_{r_{j-1}+2} \ldots x_{r_{j}}\right)^{p_{j}}}{\prod_{\substack{j=1 \\
j \neq h-1}}^{l}\left(1-z x_{1} x_{2} \ldots x_{r_{j}}\right)} d x_{1} d x_{2} \ldots d x_{m} \\
& -\int_{[0,1]^{m}} \frac{\prod_{j=1}^{l}\left(x_{r_{j-1}+1} x_{r_{j-1}+2} \ldots x_{r_{j}}\right)^{\prime}}{\prod_{\substack{j=1 \\
j \neq h}}^{l}\left(1-z x_{1} x_{2} \ldots x_{r_{j}}\right)} d x_{1} d x_{2} \ldots d x_{m},
\end{aligned}
$$

where $p_{j}^{\prime}=p_{j}$ for $j \neq h$ and $p_{h}^{\prime}=p_{h}-1$. By [1, Lemma 2] we write this equality as

$$
\begin{aligned}
I\left(p_{1}, p_{2}, \ldots, p_{h}, \ldots, p_{l}\right) & \\
= & I\left(p_{1}, p_{2}, \ldots, p_{h}-1, \ldots, p_{l}\right) \\
+ & \sum_{n_{1} \geq n_{2} \geq \cdots \geq n_{l-1} \geq 1} z^{n_{1}-1} \prod_{j=1}^{h-2} \frac{1}{\left(n_{j}+p_{j}\right)^{u_{j}}} \\
& \times \frac{1}{\left(n_{h-1}+p_{h-1}\right)^{u_{h-1}}\left(n_{h-1}+p_{h}\right)^{u_{h}}} \cdot \prod_{j=h}^{l-1} \frac{1}{\left(n_{j}+p_{j+1}\right)^{u_{j+1}}} \\
& -\sum_{n_{1} \geq n_{2} \geq \cdots \geq n_{l-1} \geq 1} z^{n_{1}-1} \prod_{j=1}^{h-1} \frac{1}{\left(n_{j}+p_{j}\right)^{u_{j}}} \\
& \quad \times \frac{1}{\left(n_{h}+p_{h}-1\right)^{u_{h}}\left(n_{h}+p_{h+1}\right)^{u_{h+1}}} \cdot \prod_{j=h+1}^{l-1} \frac{1}{\left(n_{j}+p_{j+1}\right)^{u_{j+1}}}
\end{aligned}
$$

If $h=l$, the subtracted sum reads as

$$
\frac{1}{p_{l}^{u_{l}}} \sum_{n_{1} \geq n_{2} \geq \cdots \geq n_{l-1} \geq 1} z^{n_{1}-1} \prod_{j=1}^{l-1} \frac{1}{\left(n_{j}+p_{j}\right)^{u_{j}}}
$$

Now we consider in detail the sum (7). If $p_{h-1}=p_{h}$, then

$$
\frac{1}{\left(n_{h-1}+p_{h-1}\right)^{u_{h-1}}\left(n_{h-1}+p_{h}\right)^{u_{h}}}=\frac{1}{\left(n_{h-1}+p_{h-1}\right)^{u_{h-1}+u_{h}}}
$$


i.e. the sum (77) is elementary and it is possible to apply the induction hypothesis to it. In this case the heights of polynomials $P_{\vec{t}}(z)$ in the sum decomposition (into a linear form) do not exceed

$$
(l-1) ! \cdot\left(m 2^{m}\right)^{l-2} P^{l-1},
$$

and the common denominator of coefficients of $P_{\vec{t}}(z)$ divides $D_{P}^{m-w(\vec{t})}$. If $p_{h-1} \neq p_{h}$, then we take the following partial fraction decomposition:

$$
\begin{gathered}
\frac{1}{\left(n_{h-1}+p_{h-1}\right)^{u_{h-1}}\left(n_{h-1}+p_{h}\right)^{u_{h}}}=\sum_{k=1}^{u_{h-1}} \frac{A_{k}}{\left(n_{h-1}+p_{h-1}\right)^{k}}+\sum_{k=1}^{u_{h}} \frac{B_{k}}{\left(n_{h-1}+p_{h}\right)^{k}}, \\
A_{k}=(-1)^{u_{h-1}-k}\left(\begin{array}{c}
u_{h-1}+u_{h}-k-1 \\
u_{h-1}-k
\end{array}\right) \frac{1}{\left(p_{h}-p_{h-1}\right)^{u_{h-1}+u_{h}-k}}, \\
B_{k}=(-1)^{u_{h}-k}\left(\begin{array}{c}
u_{h-1}+u_{h}-k-1 \\
u_{h}-k
\end{array}\right) \frac{1}{\left(p_{h-1}-p_{h}\right)^{u_{h-1}+u_{h}-k}} .
\end{gathered}
$$

Substituting this equality into (7), we write (7) as the sum of $u_{h-1}+u_{h}$ elementary sums (with coefficients $A_{k}$ and $B_{k}$ ). We can apply the induction hypothesis for each of them. Consider one of them,

$$
\sum_{n_{1} \geq n_{2} \geq \cdots \geq n_{l-1} \geq 1} z^{n_{1}-1} \prod_{j=1}^{h-2} \frac{1}{\left(n_{j}+p_{j}\right)^{u_{j}}} \cdot \frac{1}{\left(n_{h-1}+p_{h-1}\right)^{k}} \cdot \prod_{j=h}^{l-1} \frac{1}{\left(n_{j}+p_{j+1}\right)^{u_{j+1}}} .
$$

The corresponding parameters in it are

$l^{\prime}=l-1, \quad m^{\prime}=m+k-u_{h-1}-u_{h}, \quad \vec{p}^{\prime}=\left(p_{1}, \ldots, p_{h-2}, p_{h-1}, p_{h+1}, \ldots, p_{l}\right)$.

Let $P_{\vec{t}}(z)$ be the polynomials in the decomposition into a linear form in generalized polylogarithms; then the common denominator of the coefficients of $P_{\vec{t}}(z)$ divides $D_{P}^{m^{\prime}-w(\vec{t})}$. Since $D_{P}^{u_{h-1}+u_{h}-k} A_{k} \in \mathbb{Z}$, we have $D_{P}^{m-w(\vec{t})}\left(A_{k}\right.$. $\left.P_{\vec{t}}(z)\right) \in \mathbb{Z}[z]$ as required. The heights of $P_{\vec{t}}(z)$ do not exceed

$$
(l-1) ! \cdot\left(m 2^{m}\right)^{l-2} \cdot P^{l-1} .
$$

Consequently, the heights of the polynomials in the decomposition of sum (7) do not exceed

$$
\left(\sum_{k=1}^{u_{h-1}}\left|A_{k}\right|+\sum_{k=1}^{u_{h}}\left|B_{k}\right|\right) \cdot(l-1) ! \cdot\left(m 2^{m}\right)^{l-2} \cdot P^{l-1}
$$




$$
\begin{aligned}
& \leq\left(\sum_{k=1}^{u_{h-1}}\left(\begin{array}{c}
u_{h-1}+u_{h}-k-1 \\
u_{h-1}-k
\end{array}\right)+\sum_{k=1}^{u_{h}}\left(\begin{array}{c}
u_{h-1}+u_{h}-k-1 \\
u_{h}-k
\end{array}\right)\right) \\
& \quad \times(l-1) ! \cdot\left(m 2^{m}\right)^{l-2} \cdot P^{l-1} \\
& \leq\left(u_{h-1}+u_{h}\right) 2^{u_{h-1}+u_{h}-2} \cdot(l-1) ! \cdot\left(m 2^{m}\right)^{l-2} \cdot P^{l-1} \\
& \leq m 2^{m-2} \cdot(l-1) ! \cdot\left(m 2^{m}\right)^{l-2} \cdot P^{l-1} \\
& \leq \frac{1}{2} \cdot(l-1) ! \cdot\left(m 2^{m} P\right)^{l-1} .
\end{aligned}
$$

Sum (8) is considered similarly. Further, we can apply the induction hypothesis to the integral $I\left(p_{1}, p_{2}, \ldots, p_{h}-1, \ldots, p_{l}\right)$. For all three summands (6) , (7) , (88), denominators of the coefficients of the polynomial coefficients of $\operatorname{Le}_{\vec{t}}(z)$ in the linear form (1) divide $D_{P}^{m-w(\vec{t})}$. The heights of the polynomials $P_{\vec{s}}(z)$ for the initial sum, in case of $\sum_{j=1}^{l} p_{j}>1$, do not exceed

$$
\begin{aligned}
\left(\sum_{j=1}^{l} p_{j}-1\right) \cdot(l-1) ! \cdot\left(m 2^{m} P\right)^{l-1}+2 \cdot \frac{1}{2} & \cdot(l-1) ! \cdot\left(m 2^{m} P\right)^{l-1} \\
& =\sum_{j=1}^{l} p_{j} \cdot(l-1) ! \cdot\left(m 2^{m} P\right)^{l-1}
\end{aligned}
$$

If $\sum_{j=1}^{l} p_{j}=1$, vectors of the generalized polylogarithms from the decomposition of (7) and (8) have length less than $l$, and in the decomposition $I\left(\{0\}_{l}\right)$ there is exactly one polylogarithm of length $l$, i.e. the sets of the polylogaritms are not intersected and the estimate on the heights in this case is also valid.

It remains to prove the statement of lemma for the integral

$$
I\left(p_{1}, 0, \ldots, 0\right)=\int_{[0,1]^{m}} \frac{\left(x_{1} x_{2} \ldots x_{r_{1}}\right)^{p_{1}}}{\prod_{j=1}^{l}\left(1-z x_{1} x_{2} \ldots x_{r_{j}}\right)} d x_{1} d x_{2} \ldots d x_{m} .
$$

From the equality

$$
\left(x_{1} x_{2} \ldots x_{r_{1}}\right)^{p_{1}}=z^{-1}\left(x_{1} x_{2} \ldots x_{r_{1}}\right)^{p_{1}-1}-z^{-1}\left(x_{1} x_{2} \ldots x_{r_{1}}\right)^{p_{1}-1}\left(1-z x_{1} x_{2} \ldots x_{r_{1}}\right)
$$

it follows that

$$
I\left(p_{1}, 0, \ldots, 0\right)=z^{-1} I\left(p_{1}-1,0, \ldots, 0\right)
$$




$$
\begin{aligned}
& -z^{-1} \int_{[0,1]^{m}} \frac{\left(x_{1} x_{2} \ldots x_{r_{1}}\right)^{p_{1}-1}}{\prod_{j=2}^{l}\left(1-z x_{1} x_{2} \ldots x_{r_{j}}\right)} d x_{1} d x_{2} \ldots d x_{m} \\
= & z^{-1} I\left(p_{1}-1,0, \ldots, 0\right) \\
& -z^{-1} \sum_{n_{1} \geq \cdots \geq n_{l-1} \geq 1} z^{n_{1}-1} \frac{1}{\left(n_{1}+p_{1}-1\right)^{u_{1}} n_{1}^{u_{2}}} \prod_{j=2}^{l-1} \frac{1}{n_{j}^{u_{j+1}}},
\end{aligned}
$$

Thus, one can proceed as before in the case $p_{h}>0$ for $h>1$. Now the lemma is completely proved.

\section{Denominators of linear form coefficients}

Let us study denominators of the coefficients of the linear form. We shall use the notion of integer-valued polynomial. For a polynomial of degree $N$ to be integer-valued it is sufficient that it possesses integer values at $N+1$ neighbour integer points (see [8, Theorem 12.1]).

Let $\Delta$ be a fixed nonnegative integer. We say that a rational function $R(x)$ is $\Delta$-normal if it can be represented as

$$
R(x)=\sum_{\alpha \in \mathcal{A}} \sum_{m=1}^{M} \frac{A_{m, \alpha}}{(x+\alpha)^{m}}+P(x),
$$

where $\mathcal{A}$ is a set of nonnegative integers from a certain segment $\left[\alpha_{1}, \alpha_{2}\right]$, $D_{\Delta}^{M-m} A_{m, \alpha} \in \mathbb{Z}$ and $D_{\Delta}^{M} P(x)$ is an integer-valued polynomial.

Lemma 2 Multiplying $\Delta$-normal function by an integer-valued polynomial of degree $\leq \Delta$ remains it $\Delta$-normal.

Proof. An integer-valued polynomial $D_{\Delta}^{M} P(x)$, multiplied by another integervalued polynomial, remains the integer-valued. The statement of the lemma would be proved if we demonstrate it for

$$
R(x)=\frac{A_{m, \alpha}}{(x+\alpha)^{m}}, \quad D_{\Delta}^{M-m} A_{m, \alpha} \in \mathbb{Z} .
$$

It is carried out by induction on $m$. We check firstly the induction base $m=1$. 
Let $T(x)$ be an integer-valued polynomial of degree $\leq \Delta$ and $\alpha$ be an integer. Then

$$
\frac{T(x)}{x+\alpha}=\frac{T(-\alpha)}{x+\alpha}+Q(x),
$$

where $Q(x)$ is a polynomial of degree $\leq \Delta-1$ (if $\Delta=0$ it is absent). By the hypothesis, $T(-\alpha)$ is an integer. Consider $Q(x)$ at the points $x=-\alpha+k$, where $k=1,2, \ldots, \Delta$ :

$$
Q(-\alpha+k)=\frac{T(-\alpha+k)-T(-\alpha)}{k} .
$$

Multiplying all these numbers by $D_{\Delta}$ gives integers, hence $D_{\Delta} Q(x)$ is an integer-valued polynomial.

Thus, if $m=1$,

$$
R(x) T(x)=\frac{A_{1, \alpha} T(-\alpha)}{x+\alpha}+A_{1, \alpha} Q(x) .
$$

In addition,

$$
D_{\Delta}^{M-1}\left(A_{1, \alpha} \cdot T(-\alpha)\right)=\left(D_{\Delta}^{M-1} \cdot A_{1, \alpha}\right) T(-\alpha) \in \mathbb{Z}
$$

and

$$
D_{\Delta}^{M}\left(A_{1, \alpha} \cdot Q(x)\right)=\left(D_{\Delta}^{M-1} \cdot A_{1, \alpha}\right) \cdot\left(D_{\Delta} \cdot Q(x)\right)
$$

is an integer-valued polynomial.

Suppose that $m>1$. Then

$$
R(x) T(x)=\frac{A_{m, \alpha}}{(x+\alpha)^{m-1}} \cdot \frac{T(x)}{x+\alpha}=\frac{A_{m, \alpha} T(-\alpha)}{(x+\alpha)^{m}}+\frac{A_{m, \alpha}}{(x+\alpha)^{m-1}} Q(x) .
$$

Since

$$
D_{\Delta}^{M-m} \cdot\left(A_{m, \alpha} T(-\alpha)\right)=\left(D_{\Delta}^{M-m} \cdot A_{m, \alpha}\right) T(-\alpha) \in \mathbb{Z},
$$

the first summand is $\Delta$-normal. Write the second summand as

$$
\frac{A_{m, \alpha} / D_{\Delta}}{(x+\alpha)^{m-1}} \cdot\left(D_{\Delta} Q(x)\right) \text {. }
$$

Since $D_{\Delta}^{M-(m-1)} \cdot\left(A_{m, \alpha} / D_{\Delta}\right) \in \mathbb{Z}$ and $D_{\Delta} Q(x)$ is an integer-valued polynomial, we can apply the induction hypothesis to the latter expression. The lemma is proved. 
Define the index of a rational function $R(x)=\frac{P(x)}{Q(x)}$ as $I(R)=\operatorname{deg} P-$ $\operatorname{deg} Q$.

Lemma 3 Suppose that for the sum

$$
\mathcal{F}=\sum_{n_{1}=1}^{\infty} z^{n_{1}-1} R_{1}\left(n_{1}\right) \sum_{n_{2}=1}^{n_{1}} R_{2}\left(n_{2}\right) \cdots \sum_{n_{l}=1}^{n_{l-1}} R_{l}\left(n_{l}\right),
$$

the following inequalities are valid:

$$
\sum_{j=1}^{j_{1}}\left(I\left(R_{j}\right)+1\right) \leq 0, \quad \sum_{j=j_{1}}^{j_{2}}\left(I\left(R_{j}\right)+1\right) \leq \Delta
$$

for any $1 \leq j_{1} \leq j_{2} \leq l$, and that the functions $R_{j}$ are $\Delta$-normal. Then $\mathcal{F}$ is expressed as a finite sum $\sum_{i} \lambda_{i} \mathcal{F}_{i}, \lambda_{i} \in \mathbb{Q}$, where

$$
\mathcal{F}_{i}=\sum_{n_{1}=1}^{\infty} z^{n_{1}-1} R_{i, 1}\left(n_{1}\right) \sum_{n_{2}=1}^{n_{1}} R_{i, 2}\left(n_{2}\right) \cdots \sum_{n_{l(i)}=1}^{n_{l(i)-1}} R_{i, l(i)}\left(n_{l(i)}\right),
$$

and $I\left(R_{i, j}\right)<0$ for any $i, j$. In addition, the functions $R_{i, j}$ are $\Delta$-normal and $D_{\Delta}^{w_{i}} \lambda_{i} \in \mathbb{Z}$, where

$$
w_{i}=\sum_{j=1}^{l} M_{j}-\sum_{j=1}^{l(i)} M_{i, j},
$$

$M_{j}, M_{i, j}$ is the maximal order of poles of the functions $R_{j}$ and $R_{i, j}$.

Proof. We proceed by induction on the vector $(l, k)$, where $k$ is the number of the functions $R_{j}$ with $I\left(R_{j}\right) \geq 0(0 \leq k<l)$. Order vectors $(l, k)$ in lexicographic ally. The induction base $l=1$ is clear, since $I\left(R_{1}\right) \leq-1$ in this case by the hypothesis. We prove the statement for a vector $(l, k)$ assuming that for smaller vectors it is already proved. If $k=0$, it is nothing to prove, since $I\left(R_{j}\right)<0$ for any $j$. Let $k>0$, i.e. there exists $j$ such that $I\left(R_{j}\right) \geq 0$. The condition $I\left(R_{1}\right) \leq-1$ implies $j>1$. Expressing $R_{j}$ as the sum of a polynomial and a proper fraction, write $\mathcal{F}$ as the sum of two summands. The summand with with the proper fraction $(\Delta$-normal) has the 
number $k$ smaller by one, hence we can apply the induction base to it. Now consider the second summand, in which $R_{j}(x)=P(x)$ is a polynomial. From the normality of $R_{j}$, it follows that the polynomial $D_{\Delta}^{M_{j}} P$ is integer-valued and, in addition, the sum of the maximal orders of poles of the functions $R_{j}$ is just smaller by $M_{j}$ compared with $\mathcal{F}$.

a) If $j=l$ summation of the latter gives

$$
\sum_{n_{l}=1}^{n_{l-1}} P\left(n_{l}\right)=Q\left(n_{l-1}\right)
$$

where $D_{\Delta}^{M_{j}} Q$ is an integer-valued polynomial of degree $\operatorname{deg} P+1$. Thereby, $R_{l-1}$ is multiplied by $Q$. Thus, compared with the initial sum, the number of summations is decreased by one. We can apply the induction hypothesis to the above sum, multiplied by $D_{\Delta}^{M_{j}}$, since the index vector of involved rational functions equals $\left.\left(I\left(R_{1}\right), \ldots, I\left(R_{l-2}\right), I\left(R_{l-1}\right)+I\left(R_{l}\right)+1\right)\right)$, and multiplying by $D_{\Delta}^{M_{j}} Q(x)$ of the function $R_{l-1}$ remains it $P$-normal by Lemma 2 , because of condition (9) for $j_{1}=j_{2}=j$ we have

$$
\operatorname{deg} Q(x)=\operatorname{deg} P+1=I\left(R_{j}\right)+1 \leq \Delta .
$$

b) Suppose that $R_{j}(x)=P(x)$ for $1<j<l$. Write the initial sum as

$$
\sum_{n_{1}=1}^{\infty} z^{n_{1}-1} R_{1}\left(n_{1}\right) \sum_{n_{2}=1}^{n_{1}} R_{2}\left(n_{2}\right) \cdots \sum_{n_{j-1}=1}^{n_{j-2}} R_{j-1}\left(n_{j-1}\right) \sum_{n_{j}=1}^{n_{j-1}} P\left(n_{j}\right) \sum_{n_{j+1}=1}^{n_{j}} f\left(n_{j+1}\right),
$$

where

$$
f\left(n_{j+1}\right)=R\left(n_{j+1}\right) \sum_{n_{j+2}=1}^{n_{j+1}} R_{j+2}\left(n_{j+2}\right) \cdots \sum_{n_{l}=1}^{n_{l-1}} R_{l}\left(n_{l}\right)
$$

We have equalities:

$$
\begin{aligned}
& \sum_{n_{j}=1}^{n_{j-1}} P\left(n_{j}\right) \sum_{n_{j+1}=1}^{n_{j}} f\left(n_{j+1}\right) \\
& =\sum_{n_{j}=1}^{n_{j-1}} P\left(n_{j}\right) \sum_{n_{j+1}=1}^{n_{j-1}} f\left(n_{j+1}\right)-\sum_{n_{j}=1}^{n_{j-1}} P\left(n_{j}\right) \sum_{n_{j+1}=n_{j}+1}^{n_{j-1}} f\left(n_{j+1}\right)
\end{aligned}
$$




$$
\begin{aligned}
& =Q_{1}\left(n_{j-1}\right) \sum_{n_{j+1}=1}^{n_{j-1}} f\left(n_{j+1}\right)-\sum_{n_{j+1}=2}^{n_{j-1}} f\left(n_{j+1}\right) \sum_{n_{j}=1}^{n_{j+1}-1} P\left(n_{j}\right) \\
& =Q_{1}\left(n_{j-1}\right) \sum_{n_{j+1}=1}^{n_{j-1}} f\left(n_{j+1}\right)-\sum_{n_{j+1}=1}^{n_{j-1}} Q_{2}\left(n_{j+1}\right) f\left(n_{j+1}\right) ;
\end{aligned}
$$

in addition, $\operatorname{deg} Q_{1}=\operatorname{deg} Q_{2}=\operatorname{deg} P+1, Q_{2}(1)=0$. Thereby, we express the initial sum as the difference of sums with a smaller repetition factor, corresponding to the vectors

$$
\begin{aligned}
& \left(I\left(R_{1}\right), \ldots, I\left(R_{j-1}\right)+I\left(R_{j}\right)+1, I\left(R_{j+1}\right), \ldots, I\left(R_{l}\right)\right), \\
& \left(I\left(R_{1}\right), \ldots, I\left(R_{j-1}\right), I\left(R_{j+1}\right)+I\left(R_{j}\right)+1, \ldots, I\left(R_{l}\right)\right) .
\end{aligned}
$$

The inequality (9) is also valid for each sum. Since $D_{\Delta}^{M_{j}} P(x)$ is an integervalued polynomial and $Q_{1}(x), Q_{2}(x)$ are polynomials such that $Q_{1}(n)=$ $\sum_{k=1}^{n} P(k), Q_{2}(n)=\sum_{k=1}^{n-1} P(k)$ for every integer $n \geq 1$, it follows that $D_{\Delta}^{M_{j}} Q_{1}$ and $D_{\Delta}^{M_{j}} Q_{2}$ are integer-valued. Multiplying functions $R_{j-1}$ and $R_{j+1}$ by $D_{\Delta}^{M_{j}} Q_{1}(x)$ and $D_{\Delta}^{M_{j}} Q_{2}(x)$ remains them $P$-normal by Lemma 2 , because of

$$
\operatorname{deg} Q_{1}(x)=\operatorname{deg} Q_{2}(x)=\operatorname{deg} P+1=I\left(R_{j}\right)+1 \leq \Delta .
$$

The last inequality is due to condition (9) for $j_{1}=j_{2}=j$. Thus, we can apply the induction hypothesis for each of two sums, multiplied by $D_{\Delta}^{M_{j}}$. This completes the proof.

Lemma 4 Let the parameters $a_{i}, b_{i}, c_{j}$ be integer, $b_{i}>a_{i} \geq 1$ for $i=$ $1, \ldots, m, P=\max _{1 \leq i \leq m} b_{i}-2, q_{j}=\sum_{i=r_{j-1}+1}^{r_{j}}\left(b_{i}-a_{i}\right)$ and let inequalities $1 \leq c_{j} \leq P+1, c_{1}+\cdots+c_{j} \leq q_{1}+\cdots+q_{j}, j=1, \ldots, l ; c_{j_{1}-1}+\sum_{j=j_{1}}^{j_{2}}\left(c_{j}-q_{j}\right) \leq$ $P+1,1<j_{1} \leq j_{2} \leq l$ be valid. Suppose that $P_{\vec{s}}$ are the polynomials from the linear form $S(z)=\sum_{\vec{s}} P_{\vec{s}}\left(z^{-1}\right) \operatorname{Le}_{\vec{s}}(z)$. Then the polynomial $D_{P}^{m-w(\vec{s})} P_{\vec{s}}(z)$ has integer coefficients.

Proof. Using [1, Lemma 2], represent the integral $S(z)$ as

$$
S(z)=\frac{\prod_{i=1}^{m} \Gamma\left(b_{i}-a_{i}\right)}{\prod_{j=1}^{l} \Gamma\left(c_{j}\right)} \sum_{n_{1}=1}^{\infty} \sum_{n_{2}=1}^{n_{1}} \cdots \sum_{n_{l}=1}^{n_{l-1}} z^{n_{1}-1}
$$




$$
\times \frac{\prod_{j=1}^{l}\left[\left(n_{j}-n_{j+1}+1\right)\left(n_{j}-n_{j+1}+2\right) \ldots\left(n_{j}-n_{j+1}+c_{j}-1\right)\right]}{\prod_{j=1}^{l} \prod_{i=r_{j-1}+1}^{r_{j}}\left[\left(n_{j}+a_{i}-1\right)\left(n_{j}+a_{i}\right) \cdots\left(n_{j}+b_{i}-2\right)\right]},
$$

letting $n_{l+1} \equiv 1$. From the known formula

$$
\begin{aligned}
(x-y+1) \cdots(x-y+n)=\sum_{k=0}^{n}(-1)^{k}\left(\begin{array}{l}
n \\
k
\end{array}\right)(x+k+1) \cdots(x+n) & \\
& \times(y+1) \cdots(y+k-1)
\end{aligned}
$$

(see, for instance, [2, Lemma 5]) it follows that

$$
\begin{aligned}
& \left(n_{j}-n_{j+1}+1\right) \cdots\left(n_{j}-n_{j+1}+c_{j}-1\right)=\sum_{k_{j}=0}^{c_{j}-1}(-1)^{k_{j}}\left(\begin{array}{c}
c_{j}-1 \\
k_{j}
\end{array}\right) \\
& \times\left(n_{j}+k_{j}+1\right)\left(n_{j}+k_{j}+2\right) \cdots\left(n_{j}+c_{j}-1\right) \cdot n_{j+1}\left(n_{j+1}+1\right) \cdots\left(n_{j+1}+k_{j}-1\right) .
\end{aligned}
$$

Using this equality for each $j$, we express $S(z)$ as a linear combination with integer coefficients of sums (with fixed $k_{j}$ ) of type

$$
\begin{aligned}
\sum_{n_{1}=1}^{\infty} \sum_{n_{2}=1}^{n_{1}} \cdots \sum_{n_{l}=1}^{n_{l-1}} z^{n_{1}-1} \prod_{j=1}^{l} & p_{j}^{1}\left(n_{j}\right) p_{j}^{2}\left(n_{j+1}\right) \\
& \times \prod_{i=r_{j-1}+1}^{r_{j}} \frac{\Gamma\left(b_{i}-a_{i}\right)}{\left(n_{j}+a_{i}-1\right)\left(n_{j}+a_{i}\right) \cdots\left(n_{j}+b_{i}-2\right)}
\end{aligned}
$$

where

$$
\begin{aligned}
p_{j}^{1}(x) & =\frac{\left(x+k_{j}+1\right)\left(x+k_{j}+2\right) \cdots\left(x+c_{j}-1\right)}{\left(c_{j}-k_{j}-1\right) !}, \\
p_{j}^{2}(x) & =\frac{x(x+1) \cdots\left(x+k_{j}-1\right)}{k_{j} !}
\end{aligned}
$$

are integer-valued polynomials. Write the last expression as

$$
\sum_{n_{1}=1}^{\infty} z^{n_{1}-1} R_{1}\left(n_{1}\right) \sum_{n_{2}=1}^{n_{1}} R_{2}\left(n_{2}\right) \cdots \sum_{n_{l}=1}^{n_{l-1}} R_{l}\left(n_{l}\right) .
$$

where

$$
R_{j}(x)=p_{j}^{1}(x) p_{j-1}^{2}(x) \prod_{i=r_{j-1}+1}^{r_{j}} \frac{\Gamma\left(b_{i}-a_{i}\right)}{\left(x+a_{i}-1\right)\left(x+a_{i}\right) \cdots\left(x+b_{i}-2\right)} .
$$


For $j=1$, define $p_{0}^{2}(x) \equiv 1, c_{0}=1, k_{0}=0$.

Since $\left|\left(b_{i_{1}}-2\right)-\left(a_{i_{2}}-1\right)\right| \leq P-\left(\min _{1 \leq i \leq m} a_{i}-1\right) \leq P$, the product

$$
\prod_{i=r_{j-1}+1}^{r_{j}} \frac{\Gamma\left(b_{i}-a_{i}\right)}{\left(x+a_{i}-1\right)\left(x+a_{i}\right) \cdots\left(x+b_{i}-2\right)}
$$

is $P$-normal. Consequently, we can apply Lemma 2 to it multiplied by $p_{j}^{1}(x)$ and $p_{j-1}^{2}(x)$. The estimate on polynomial degrees are valid: $\operatorname{deg} p_{j}^{1} \leq c_{j}-1 \leq$ $P$ and $\operatorname{deg} p_{j-1}^{2} \leq c_{j-1}-1 \leq P$. Thus, $R_{j}$ is $P$-normal function.

Verify condition (9) for the function $R_{j}$ :

$$
\begin{aligned}
\sum_{j=j_{1}}^{j_{2}}\left(I\left(R_{j}\right)+1\right) & =\sum_{j=j_{1}}^{j_{2}}\left(k_{j-1}+\left(c_{j}-k_{j}-1\right)-q_{j}+1\right) \\
& \leq c_{j_{1}-1}-1+\sum_{j=j_{1}}^{j_{2}}\left(c_{j}-q_{j}\right) \leq P .
\end{aligned}
$$

The last inequality holds by the hypothesis of the lemma.

Using Lemma 3, we may assume that $I\left(R_{j}\right)<0$ for any $j$, and $R_{j}$ is $P$ normal. Equivalently, we expressed $S(z)$ as a linear combination with integer coefficients of sums

$$
\sum_{n_{1}=1}^{\infty} z^{n_{1}-1} \frac{A_{\vec{u}, \vec{\alpha}}}{\left(n_{1}+\alpha_{1}\right)^{u_{1}}} \sum_{n_{2}=1}^{n_{1}} \frac{1}{\left(n_{2}+\alpha_{2}\right)^{u_{2}}} \cdots \sum_{n_{l^{\prime}}=1}^{n_{l^{\prime}-1}} \frac{1}{\left(n_{l^{\prime}}+\alpha_{l^{\prime}}\right)^{u_{l^{\prime}}}},
$$

where $l^{\prime} \leq l, p \leq \alpha_{j} \leq P$. Herewith $D_{P}^{m-w(\vec{u})} A_{\vec{u}, \vec{\alpha}} \in \mathbb{Z}$. Furthermore, for a polynomial $P_{\vec{s}}$ in the decomposition of the elementary sum

$$
\sum_{n_{1}=1}^{\infty} z^{n_{1}-1} \frac{1}{\left(n_{1}+\alpha_{1}\right)^{u_{1}}} \sum_{n_{2}=1}^{n_{1}} \frac{1}{\left(n_{2}+\alpha_{2}\right)^{u_{2}}} \cdots \sum_{n_{l^{\prime}}=1}^{n_{l^{\prime}-1}} \frac{1}{\left(n_{l^{\prime}}+\alpha_{l^{\prime}}\right)^{u_{l^{\prime}}}}
$$

into the linear form $\sum_{\vec{s}} P_{\vec{s}}\left(z^{-1}\right) \operatorname{Le}_{\vec{s}}(z)$, we have the inclusion $D_{P}^{w(\vec{u})-w(\vec{s})} P_{\vec{s}}(z) \in$ $\mathbb{Z}[z]$ by Lemma 1, This implies the lemma.

Remark. Lemma 4 remains valid if some of $c_{j}$ are equal to zero. 
Theorem 1 Let the parameters $a_{i}, b_{i}, c_{j}$ be integers, $b_{i}>a_{i} \geq 1$ for $i=$ $1, \ldots, m$ and $c_{j} \geq 1, c_{1}+\cdots+c_{j} \leq q_{1}+\cdots+q_{j}$, where $q_{j}=\sum_{i=r_{j-1}+1}^{r_{j}}\left(b_{i}-a_{i}\right)$, $j=1, \ldots, l$; let $d_{j}$ be nonnegative integers, satisfying $d_{j} \leq c_{j}$ for $j=1, \ldots, l$ and $\sum_{k=j}^{l} d_{k}<a_{i}$ for $j=1, \ldots, l$ and $r_{j-1}<i \leq r_{j}$. Denote

$$
\Delta=\max _{1 \leq j \leq l} \max _{r_{j-1}<i \leq r_{j}}\left(b_{i}-\sum_{k=j}^{l} d_{k}-2\right) .
$$

Assume that the following inequalities are valid: $1 \leq c_{j} \leq \Delta+1, c_{j_{1}-1}+$ $\sum_{j=j_{1}}^{j_{2}}\left(c_{j}-q_{j}\right) \leq \Delta+1,1<j_{1} \leq j_{2} \leq l$, and that $P_{\vec{s}}$ are the polynomials from the linear form $S(z)=\sum_{\vec{s}} P_{\vec{s}}\left(z^{-1}\right) \operatorname{Le}_{\vec{s}}(z)$. Then the polynomial $D_{\Delta}^{m-w(\vec{s})} P_{\vec{s}}(z)$ has integer coefficients.

Proof. Expand the integrand of $S(z)$ using the following equalities:

$$
\left(x_{1} x_{2} \cdots x_{r_{j}}\right)^{d_{j}}=\left(\frac{1-\left(1-z x_{1} x_{2} \cdots x_{r_{j}}\right)}{z}\right)^{d_{j}}, \quad j=1, \ldots, l .
$$

It is possible to do, since $\sum_{k=j}^{l} d_{k}<a_{i}$ for $j=1, \ldots, l$ and $r_{j-1}<i \leq r_{j}$. This results in a linear combination with integer coefficients of expressions of the form

$$
\frac{1}{z^{d_{1}+d_{2}+\cdots+d_{l}}} \int_{[0,1]^{m}} \frac{\prod_{i=1}^{m} x_{i}^{a_{i}^{\prime}-1}\left(1-x_{i}\right)^{b_{i}-a_{i}-1}}{\prod_{j=1}^{l}\left(1-z x_{1} x_{2} \ldots x_{r_{j}}\right)^{c_{j}^{\prime}}} d x_{1} d x_{2} \ldots d x_{m}
$$

with parameters $c_{j}^{\prime}$, satisfying $0 \leq c_{j}^{\prime} \leq c_{j}, a_{i}^{\prime}=a_{i}-\sum_{k=j}^{l} d_{k} \geq 1$ for $j=1, \ldots, l$ and $r_{j-1}<i \leq r_{j}$. Application of Lemma 4 (in the lemma, $\Delta$ appears as $P$ ) to each such integral completes the proof.

Corollary 1 Let the integral $S(z)$ has parameters

$$
a_{i}=n+1, \quad b_{i}=2 n+2, \quad c_{j}=n+1 .
$$

Then the polynomial $D_{n}^{m-w(\vec{s})} P_{\vec{s}}(z)$ has integer coefficients.

Proof. Take $d_{j}=0$ for $j=1, \ldots, l-1$ and $d_{l}=n$ in Theorem 11. Then $\Delta=n$ and all conditions of the theorem are satisfied. 
We apply Corollary 1 to the integral

$$
I_{2 l+1, n}(z)=\int_{[0,1]^{2 l+1}} \frac{\prod_{i=1}^{2 l+1} x_{i}^{n}\left(1-x_{i}\right)^{n} d x_{1} d x_{2} \ldots d x_{2 l+1}}{\prod_{j=1}^{l}\left(1-z x_{1} \ldots x_{2 j}\right)^{n+1}\left(1-z x_{1} x_{2} \ldots x_{2 l} x_{2 l+1}\right)^{n+1}} .
$$

By [1, Theorem 6],

$$
I_{2 l+1, n}(z)=\sum_{k=0}^{l} P_{k}\left(z^{-1}\right) \operatorname{Le}_{\{2\}_{k}, 1}(z)+\sum_{k=0}^{l-1} T_{k}\left(z^{-1}\right) \operatorname{Le}_{1,\{2\}_{k}, 1}(z)-U\left(z^{-1}\right),
$$

$\left(\{a\}_{k}\right.$ means $\{a, \ldots, a\}, k$ times repeated) where $P_{k}, T_{k}, U$ are polynomials with rational coefficients and $P_{0}(1)=0, T_{k}(1)=0$. From Corollary [1 we conclude that these polynomials multiplied by $D_{n}^{2 l+1}$ have integer coefficients. Letting $z \rightarrow 1-$ and using the equalities $\operatorname{Le}_{\{2\}_{k}, 1}(1)=2 \zeta(2 k+1)$ (see [7]) and (3), this proves Vasiliev's conjecture (21).

\section{Estimate of linear form coefficients}

It is important in many arithmetical applications to have upper estimates for absolute values of the linear form coefficients. In this section, we study the height of a polynomial in a linear form in generalized polylogarithms, that originates from the integral $S(z)$ (see (1) ).

We start with an estimate for factorial coefficients.

Lemma 5 For nonnegative integers a and b, the following estimate holds:

$$
\frac{1}{a+b+1} \cdot \frac{(a+b)^{a+b}}{a^{a} b^{b}} \leq \frac{(a+b) !}{a ! b !} \leq \frac{(a+b)^{a+b}}{a^{a} b^{b}}
$$

(if $x=0$, we let $x^{x}=1$ ).

Proof. If $a=0$ or $b=0$, then both inequalities are valid. In what follows, suppose that $a$ and $b$ are positive integers.

Consider the Beta-integral

$$
\int_{0}^{1} x^{a}(1-x)^{b} d x=B(a+1, b+1)=\frac{a ! b !}{(a+b+1) !} .
$$


The function $f(x)=x^{a}(1-x)^{b}$, on the segment [0,1], achives its maximum at the point $x=a /(a+b)$. Hence,

$$
\frac{a ! b !}{(a+b+1) !} \leq f\left(\frac{a}{a+b}\right)=\frac{a^{a} b^{b}}{(a+b)^{a+b}},
$$

proving the first inequality. Now we prove the second inequality by induction on the value of $a+b$. The induction base $a=b=1$ is valid. Introduce the notation

$$
g(a, b)=\frac{(a+b) !}{a ! b !}
$$

Assuming $b>1$, the induction hypothesis yields

$$
g(a, b-1) \leq \frac{(a+b-1)^{a+b-1}}{a^{a}(b-1)^{b-1}} .
$$

From the definition of the function $g$,

$$
\frac{g(a, b)}{g(a, b-1)}=\frac{a+b}{b}
$$

The function $(1+1 / m)^{m}$ monitonically increases with $m$, hence

$$
\left(1+\frac{1}{a+b-1}\right)^{a+b-1} \geq\left(1+\frac{1}{b-1}\right)^{b-1} .
$$

Write the last inequality as

$$
\frac{(a+b-1)^{a+b-1}}{(b-1)^{b-1}} \leq \frac{(a+b)^{a+b-1}}{b^{b-1}}
$$

Thus,

$$
\begin{aligned}
g(a, b)=\frac{a+b}{b} \cdot g(a, b-1) & \leq \frac{a+b}{b} \cdot \frac{(a+b-1)^{a+b-1}}{a^{a}(b-1)^{b-1}} \\
& \leq \frac{a+b}{b} \cdot \frac{(a+b)^{a+b-1}}{a^{a} b^{b-1}}=\frac{(a+b)^{a+b}}{a^{a} b^{b}}
\end{aligned}
$$

which is the required assertion.

Remark. Expression

$$
\frac{(a+b)^{a+b}}{a^{a} b^{b}}
$$


can be written as

$$
\left(\frac{(\alpha+\beta)^{\alpha+\beta}}{\alpha^{\alpha} \beta^{\beta}}\right)^{n}
$$

where $\alpha=a / n, \beta=b / n$.

By [1, Lemma 2], the integral $S(z)$ is expressed as

$$
S(z)=\sum_{n_{1} \geq n_{2} \geq \cdots \geq n_{l} \geq 1} R\left(n_{1}, n_{2}, \ldots, n_{l}\right) z^{n_{1}-1}
$$

where

$$
\begin{aligned}
R\left(\zeta_{1}, \zeta_{2}, \ldots, \zeta_{l}\right) & =\frac{\prod_{i=1}^{m} \Gamma\left(b_{i}-a_{i}\right)}{\prod_{j=1}^{l} \Gamma\left(c_{j}\right)} \\
& \times \frac{\prod_{j=1}^{l}\left[\left(\zeta_{j}-\zeta_{j+1}+1\right)\left(\zeta_{j}-\zeta_{j+1}+2\right) \ldots\left(\zeta_{j}-\zeta_{j+1}+c_{j}-1\right)\right]}{\prod_{j=1}^{l} \prod_{i=r_{j-1}+1}^{r_{j}}\left[\left(\zeta_{j}+a_{i}-1\right)\left(\zeta_{j}+a_{i}\right) \ldots\left(\zeta_{j}+b_{i}-2\right)\right]},
\end{aligned}
$$

To the end of the section, suppose that the parameters $a_{i}, b_{i}, c_{j}$ depend linearly on an increasing parameter $n$, i.e.

$a_{i}=\alpha_{i} n+\alpha_{i}^{\prime}, \quad b_{i}=\beta_{i} n+\beta_{i}^{\prime}, \quad c_{j}=\gamma_{j} n+\gamma_{j}^{\prime}, \quad \alpha_{i}, \beta_{i}, \gamma_{j} \in \mathbb{N}, \quad \alpha_{i}^{\prime}, \beta_{i}^{\prime}, \gamma_{j}^{\prime} \in \mathbb{Z}$.

As before, $q_{j}=\sum_{i=r_{j-1}+1}^{r_{j}}\left(b_{i}-a_{i}\right)$. We also use notation

$$
\begin{gathered}
p_{j}=\min _{r_{j-1}+1 \leq i \leq r_{j}} a_{i}-1, \quad P_{j}=\max _{r_{j-1}+1 \leq i \leq r_{j}} b_{i}-2, \\
h_{j}=\min _{r_{j-1}+1 \leq i \leq r_{j}} \alpha_{i}, \quad H_{j}=\max _{r_{j-1}+1 \leq i \leq r_{j}} \beta_{i} \\
\varphi(x, y)=|x+y|^{x+y} \cdot|x|^{-x}
\end{gathered}
$$

Here and in what follows, $|x|^{x}=1$ if $x=0$, that agrees with the limit value of $|x|^{x}$ as $x \rightarrow 0$.

Lemma 6 Let $c_{1} \leq q_{1}$ and $c_{j-1}+c_{j} \leq q_{j}$ for $j=2, \ldots, l$. Then

$$
R\left(\zeta_{1}, \zeta_{2}, \ldots, \zeta_{l}\right)=\sum_{\vec{s}, \vec{k}} A_{\vec{s}, \vec{k}} \prod_{j=1}^{l} \frac{1}{\left(\zeta_{j}+k_{j}\right)^{s_{j}}}
$$

and

$$
\left|A_{\vec{s}, \vec{k}}\right| \leq\left(F\left(x_{1}, \ldots, x_{l}\right)\right)^{n+o(n)}, \quad n \rightarrow \infty
$$


where

$$
x_{j}=\frac{k_{j}-p_{j}}{P_{j}-p_{j}} \in[0,1]
$$

and

$$
\begin{aligned}
F\left(x_{1}, \ldots, x_{l}\right)= & \prod_{j=1}^{l} \prod_{i=r_{j-1}+1}^{r_{j}} \frac{\left(\beta_{i}-\alpha_{i}\right)^{\beta_{i}-\alpha_{i}}}{\varphi\left(\alpha_{i}-h_{j}-\left(H_{j}-h_{j}\right) x_{j}, \beta_{i}-\alpha_{i}\right)} \\
& \times \prod_{j=1}^{l-1} \frac{\varphi\left(h_{j+1}+\left(H_{j+1}-h_{j+1}\right) x_{j+1}-h_{j}-\left(H_{j}-h_{j}\right) x_{j}, \gamma_{j}\right)}{\gamma_{j}^{\gamma_{j}}} \\
& \times \frac{\varphi\left(h_{l}+\left(H_{l}-h_{l}\right) x_{l}-\gamma_{l}, \gamma_{l}\right)}{\gamma_{l}^{\gamma_{l}}} .
\end{aligned}
$$

Proof. Expand the numerator of the function $R$ into the sum of monomials. Consider any monomial and the corresponding function $\widehat{R}\left(\zeta_{1}, \zeta_{2}, \ldots, \zeta_{l}\right)$. The degree of the numerator is less than the degree of the denominator in each variable in $\widehat{R}$. Hence, the function $R\left(\zeta_{1}, \zeta_{2}, \ldots, \zeta_{l}\right)$ can be represented as

$$
R\left(\zeta_{1}, \zeta_{2}, \ldots, \zeta_{l}\right)=\sum_{\vec{s}, \vec{k}} A_{\vec{s}, \vec{k}} \prod_{j=1}^{l} \frac{1}{\left(\zeta_{j}+k_{j}\right)^{s_{j}}}
$$

Let $m_{j}$ be the maximal order of the pole in variable $\zeta_{j}$. Cauchy's integral formula for a polycylindrical domain (see [9, (1.28)]), applied to partial derivatives of the function

$$
\left(\zeta_{1}+k_{1}\right)^{m_{1}} \cdots\left(\zeta_{l}+k_{l}\right)^{m_{l}} R\left(\zeta_{1}, \zeta_{2}, \ldots, \zeta_{l}\right)
$$

implies

$$
\begin{aligned}
& A_{\vec{s}, \vec{k}}=\frac{1}{(2 \pi i)^{l}} \int_{\left|\zeta_{1}+k_{1}\right|=\frac{1}{2}} \cdots \int_{\left|\zeta_{l}+k_{l}\right|=\frac{1}{2}} R\left(\zeta_{1}, \zeta_{2}, \ldots, \zeta_{l}\right) \\
& \times\left(\zeta_{1}+k_{1}\right)^{s_{1}-1} \cdots\left(\zeta_{l}+k_{l}\right)^{s_{l}-1} d \zeta_{1} \cdots d \zeta_{l} .
\end{aligned}
$$

Introduce the function

$$
\Phi(u, v)=|u+v| !^{\operatorname{sign}(u+v)} \cdot|u| !^{-\operatorname{sign}(u)},
$$


defined for integers $u$ and $v$. The following inequalities holds on the circle $\left|\zeta_{j}+k_{j}\right|=1 / 2$ :

$$
\begin{gathered}
\left|\left(\zeta_{j}+a_{i}-1\right) \cdots\left(\zeta_{j}+b_{i}-2\right)\right| \geq \Phi\left(\left(a_{i}-1\right)-k_{j}, b_{i}-a_{i}-1\right) e^{o(n)}, \\
\left|\zeta_{l}\left(\zeta_{l}+1\right) \cdots\left(\zeta_{l}+c_{l}-2\right)\right| \leq \Phi\left(k_{l}-c_{l}+1, c_{l}-1\right) e^{o(n)}, \\
\left|\left(\zeta_{j}-\zeta_{j+1}+1\right) \cdots\left(\zeta_{j}-\zeta_{j+1}+c_{j}-1\right)\right| \leq \Phi\left(k_{j+1}-k_{j}, c_{j}-1\right) e^{o(n)} .
\end{gathered}
$$

We prove only the first inequality (the latter ones are proved similarly). First, consider the case $k_{j}$ lying in the interval $\left(a_{i}-1, b_{i}-2\right)$. For $N<k_{j}$, we have

$$
\left|\zeta_{j}+N\right|=\left|\left(k_{j}-N\right)-\left(\zeta_{j}+k_{j}\right)\right| \geq\left(k_{j}-N\right)-\frac{1}{2},
$$

and, for $N>k_{j}$,

$$
\left|\zeta_{j}+N\right|=\left|\left(N-k_{j}\right)+\left(\zeta_{j}+k_{j}\right)\right| \geq\left(N-k_{j}\right)-\frac{1}{2} .
$$

Consequently (Set a product to be 1 if upper limit greater than lower),

$$
\begin{aligned}
\left|\left(\zeta_{j}+a_{i}-1\right) \cdots\left(\zeta_{j}+b_{i}-2\right)\right| & \geq \prod_{N=a_{i}-1}^{k_{j}-1}\left(k_{j}-N-\frac{1}{2}\right) \cdot \frac{1}{2} \cdot \prod_{N=k_{j}+1}^{b_{i}-2}\left(N-k_{j}-\frac{1}{2}\right) \\
& \geq \frac{1}{8} \prod_{N=a_{i}-1}^{k_{j}-2}\left(k_{j}-N-1\right) \cdot \prod_{N=k_{j}+2}^{b_{i}-2}\left(N-k_{j}-1\right) \\
& =\frac{\left(k_{j}-\left(a_{i}-1\right)\right) !\left(\left(b_{i}-2\right)-k_{j}\right) !}{8\left(k_{j}-\left(a_{i}-1\right)\right)\left(\left(b_{i}-2\right)-k_{j}\right)} \\
& =\Phi\left(\left(a_{i}-1\right)-k_{j}, b_{i}-a_{i}-1\right) e^{o(n)} .
\end{aligned}
$$

Now consider the case $k_{j}<a_{i}-1$ :

$$
\begin{aligned}
\left|\left(\zeta_{j}+a_{i}-1\right) \cdots\left(\zeta_{j}+b_{i}-2\right)\right| & \geq \prod_{N=a_{i}-1}^{b_{i}-2}\left(N-k_{j}-\frac{1}{2}\right) \geq \frac{1}{2} \prod_{N=a_{i}}^{b_{i}-2}\left(N-k_{j}-1\right) \\
& =\frac{a_{i}-1-k_{j}}{2\left(b_{i}-2-k_{j}\right)} \cdot \frac{\left(\left(b_{i}-2\right)-k_{j}\right) !}{\left(\left(a_{i}-1\right)-k_{j}\right) !} \\
& =\Phi\left(\left(a_{i}-1\right)-k_{j}, b_{i}-a_{i}-1\right) e^{o(n)} .
\end{aligned}
$$


The case $k_{j}>b_{i}-2$ is considered similarly. Cases $k_{j}=a_{i}-1$ or $k_{j}=b_{i}-2$ can be verified by direct substitution.

Thus,

$$
\begin{aligned}
\left|A_{\vec{s}, \vec{k}}\right| \leq \prod_{j=1}^{l} & \prod_{i=r_{j-1}+1}^{r_{j}} \frac{\left(b_{i}-a_{i}-1\right) !}{\Phi\left(\left(a_{i}-1\right)-k_{j}, b_{i}-a_{i}-1\right)} \\
& \times \prod_{j=1}^{l-1} \frac{\Phi\left(k_{j+1}-k_{j}, c_{j}-1\right)}{\left(c_{j}-1\right) !} \cdot \frac{\Phi\left(k_{l}-c_{l}+1, c_{l}-1\right)}{\left(c_{l}-1\right) !} \cdot e^{o(n)} .
\end{aligned}
$$

Make the change $k_{j}=p_{j}+\left(P_{j}-p_{j}\right) x_{j}, j=1, \ldots, l, x_{j} \in[0,1]$. From Lemma 5 and estimate (11) it follows that

$$
\left|A_{\vec{s}, \vec{k}}\right| \leq\left(F\left(x_{1}, \ldots, x_{l}\right)\right)^{n} e^{o(n)},
$$

where $F\left(x_{1}, \ldots, x_{l}\right)$ is the function, specified in the lemma statement.

Theorem 2 Let $c_{1} \leq q_{1}$ and $c_{j-1}+c_{j} \leq q_{j}$ for $j=2, \ldots, l$. Then the heights of all polynomials in the linear form $S(z)=\sum_{\vec{s}} P_{\vec{s}}\left(z^{-1}\right) \operatorname{Le}_{\vec{s}}(z)$ do not exceed $M^{n+o(n)}$, as $n \rightarrow \infty$, where $M$ is the maximum of function (10) on the cube $[0,1]^{l}$.

Proof. By Lemma 6, we have the equality:

$$
\begin{aligned}
S(z) & =\sum_{n_{1} \geq n_{2} \geq \cdots \geq n_{l} \geq 1} R\left(n_{1}, n_{2}, \ldots, n_{l}\right) z^{n_{1}-1} \\
& =\sum_{\vec{s}, \vec{k}} A_{\vec{s}, \vec{k}} \sum_{n_{1} \geq n_{2} \geq \cdots \geq n_{l} \geq 1} z^{n_{1}-1} \prod_{j=1}^{l} \frac{1}{\left(\zeta_{j}+k_{j}\right)^{s_{j}}} .
\end{aligned}
$$

Since $s_{j} \leq m$ and $b_{i}-a_{i} \leq C n$, then the number of summands in the external sum does not exceed $(m \cdot C n)^{l}=e^{o(n)}$. Furthermore, consider the decomposition of the elementary sum

$$
\sum_{n_{1} \geq n_{2} \geq \cdots \geq n_{l} \geq 1} z^{n_{1}-1} \prod_{j=1}^{l} \frac{1}{\left(n_{j}+k_{j}\right)^{s_{j}}}
$$

into a linear form in polylogarithms for a fixed $\vec{s}$ and $\vec{k}$. The heights of all polynomials in it is $e^{o(n)}$ by Lemma 1. From Lemma [6 it follows that $\left|A_{\vec{s}, \vec{k}}\right| \leq M^{n+o(n)}$. This implies the statement of theorem. 


\section{References}

[1] Zlobin S.A., Expanding multiple integrals into linear forms // Matem. Zametki. 2005. V. 77. No. 5. P. 683-706.

[2] VAsilyev D.V., On small linear forms for the values of the Riemann zeta-function at odd integers // Preprint No. 1 (558). Minsk: Nat. Acad. Sci. Belarus, Institute Math., 2001.

[3] Beukers F., A note on the irrationality of $\zeta(2)$ and $\zeta(3) / /$ Bull. London Math Society. 1979. V. 11. No. 3. P. 268-272.

[4] Zudilin W.V., Well-poised hypergeometric series and multiple integrals // Uspehi Matem. Nauk. 2002. V. 57. No. 4. P. 177-178.

[5] Krattenthaler C., Rivoal T., Hypergéométrie et fonction zêta de Riemann // Preprint (December 2004), submitted for publication; // http://arxiv.org/abs/math/0311114.

[6] ZLOBin S.A, Integrals expressible as linear forms in generalized polylogarithms // Matem. Zametki. 2002. V. 71. No. 5. P. 782-787.

[7] ZLOBIn S.A., Generating functions for multiple zeta values // Vestnik MGU. Ser. 1. Matem., Mekh. 2005. No. 2. P. 55-59.

[8] Prasolov V.V., Polynomials // M.: MCCME, 1999.

[9] Fuchs B.A., Introduction to the theory of analytic functions of several complex variables // M.: Gos. Izd. Fiz.-mat. Lit., 1962. 